\title{
Application of motion controller in cargo hoist
}

\author{
Hong-Yan SHI ${ }^{1, a}$, \\ ${ }^{1}$ Inner Mongolia University for the Nationalities, College of Mechanical Engineering, Tong Liao, \\ 028000, China. \\ ajdxashy@126.com
}

Keywords: Cargo hoist, Motion controller, Control system.

\begin{abstract}
Cargo hoist have important applications in daily life and production, the traditional general cargo hoist always uses relay and contactor control, this control method has the disadvantages of poor reliability and high failure rate. In view of this situation, in this paper a cargo hoist motion control system is designed based on the motion controller to lift small goods, the motion controller applied to cargo hoist can improve the system reliability, safety, and reduce the failure rate, it has a certain practical significance.
\end{abstract}

\section{Introduction}

Cargo hoist has a wide range of applications in daily life and production, especially in small goods lift, it has a great role in our daily life, such as that hotels, warehouses and other places of the freight can use cargo hoist, it can save manpower, improve the efficiency, reduce the comprehensive cost [1]. Small cargo hoist generally adopts relay and contactor control, but relay controller and hardware control circuit wiring has the disadvantages of complexity, poor reliability, it often appears fault [2-3]. Due to the complexity of the wiring, once appear mistake is very difficult to carry out the investigation. In order to change this situation the control mode of cargo hoist must be changed, in recent years, the motion controller with high control precision, fast response and so on has been widely used [4]. Based on the motion controller as the core, the cargo hoist control system is designed. The control system based on motion controller has the advantages of good flexibility, good reliability, and can overcome the traditional relay contactor control shortcomings, improve the service life of cargo hoist.

\section{Overall design of cargo hoist}

Mechanical structure of cargo hoist. As shown in Figure 1, a small cargo hoist is mainly composed of the motor, a guide wheel, steel wire rope, control switch etc. Small cargo hoist is mainly used to complete the goods on the transport, so the most basic function is to automatically rise and fall, in order to ensure the safety, it must also have an emergency stop on a failure of the function. Hoist up and down is mainly controlled by motor forwarding and reversing. The limit switch can detect the location of the warehouse, to detect and prevent the position overrun. At the same time, the system is equipped with electromagnetic brake to ensure safe operation. 


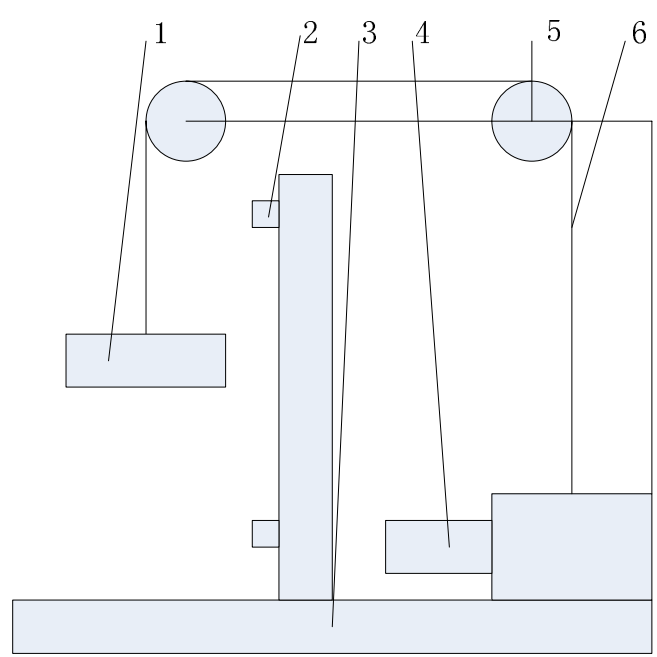

1.Warehouse 2.Limit switch 3.Frame 4.Motor

5. Guide wheel 6 . Steel wire rope

Figure 1.The cargo hoist schematic diagram

Control system design. As shown in Figure 2, the control system is mainly composed of the control panel, controller and actuator parts. There are various control switches and buttons on the panel, they are used to control the hoist motion; controller is the motion controller, the system controls the actuator through the motion controller; actuating element comprises motor, brake etc. The motion controller is the control core, the motion controller is used for receiving signals sent by control panel to control the other actuator according to the procedures.

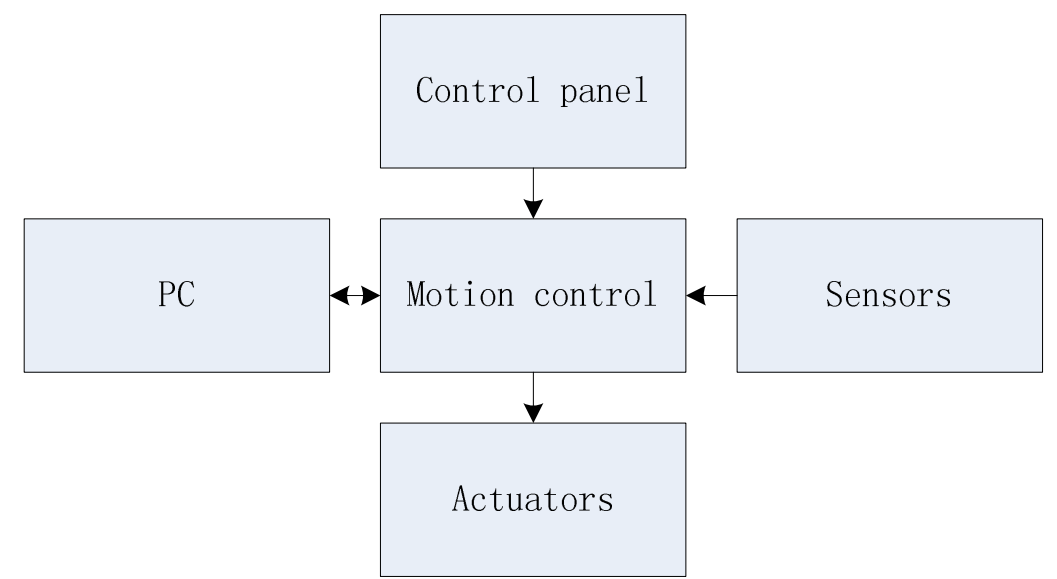

Figure 2.The cargo hoist control system

\section{Software design}

Software structure design. The motion controller does not have ability of separate programming, it is a kind of open controller based on PC. The motion controller does not have the development environment, so you need a PC to do programming and debugging of the machine. As shown in Figure 3, the upper and lower computer program is designed in this paper, $\mathrm{PC}$ as the host machine is used for the overall program planning and management; motion controller as slave machine is used to perform motion control program. It is programmed by the computer program, the written program will be transmitted to the motion controller, motion controller implements the procedures. 


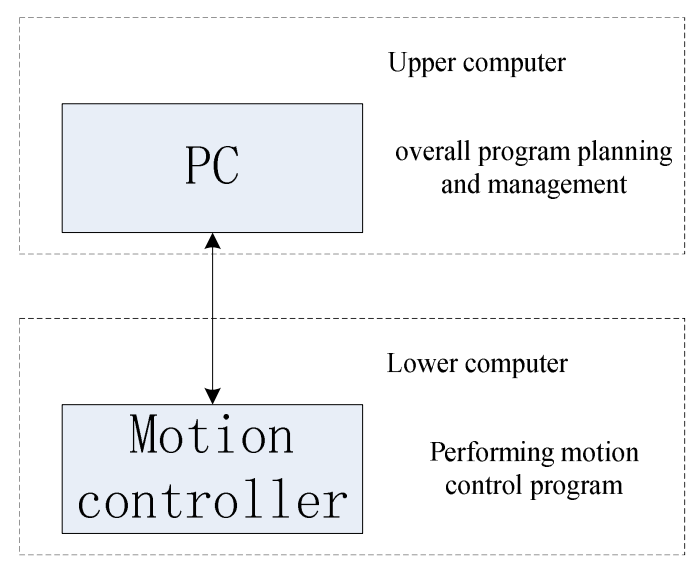

Figure3. Software structure

Module design of the software. In order to further improve flexibility and portability of the control system, the software adopts the modular design way. According to the control level, they are respectively management module, man-machine interaction module, control module and feedback module. As shown in Figure 4, the management module is mainly written in a computer program, it is used to carry out overall management; man-machine interaction module is corresponding to the source code with the buttons on the control panel; control module and feedback module is used to control the cargo hoist movement and stop, including processing fault.

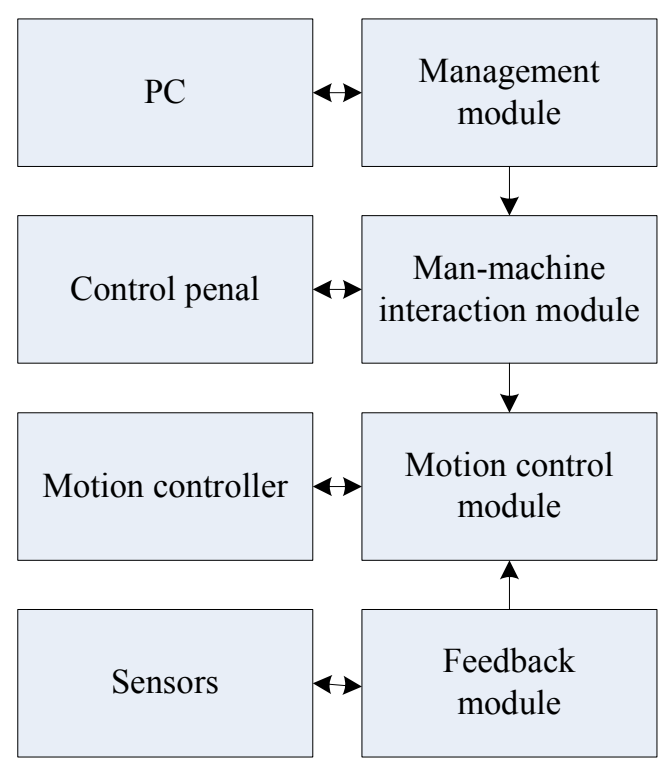

Figure4. Module design of the software

I/O design. The system sends and receives signal through the $\mathrm{I} / \mathrm{O}$ interface controller, including limit switch, relay and contactor, the system design of I/O is shown in table 1.

Table1. System I/O design

\begin{tabular}{c|c|c|c}
\hline \multicolumn{2}{|c|}{ Input } & \multicolumn{2}{c}{ Output } \\
\hline IN0 & Limit switch 1 & OUT0 & Relay 1 \\
\hline IN1 & Limit switch 2 & OUT1 & Relay 2 \\
\hline IN2 & Position switch 1 & OUT2 & Contactor 1 \\
\hline IN3 & Position switch 2 & OUT3 & Contactor 2 \\
\hline & & OUT4 & Brake \\
\hline & & OUT5 & Indicator light 1 \\
\hline & & OUT6 & Indicator light 2 \\
\hline
\end{tabular}




\section{Summary}

In view the shortcomings of the traditional cargo hoist by relay and controller as the control system, a kind of small cargo hoist is designed based on motion controller. The use of motion controller to build control system can improve the system reliability, the safety, and reduce the failure rate. This system can be used in hotel, warehouse and transport of goods between floors to replace human labor, it can improve work efficiency and reduce production costs.

\section{References}

[1] Duan Zongjun. Hoist introduction [J]. Logistics technology, 2001, (3): 9-9.

[2] Zhang Jinfeng. The application and control system of lifting machine in the warehouse [J]. Hoisting and conveying machinery, 2000, (1): 18-19.

[3] Yang Haoqiang, Sun Xiuru, Cheng Caixia. Study on small automatic lifting machine design [J]. Mining machinery, 2005, (11): 118-119.

[4] Wu Hong, Jiang Shilong, Gong Xiaoyun. Current situation and development of motion controller [J]. Manufacturing technology and machine tool, 2004, (1): 24-27. 